Introducción

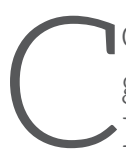
on el fin de que el Observatorio en Seguridad Alimentaria y Nutricional del Estado de Veracruz (OBSAN-UV), administrado por la Facultad de Nutrición-Xalapa, de la Universidad Veracruzana, continúe siendo una fuente de información confiable para los ámbitos gubernamentales, académico y de investigación se debe actualizar de manera permanente y divulgar los resultados más relevantes.

En esta ocasión se presentan algunos indicadores que se han incluido a la base de datos y su análisis desde diversos puntos de vista (per cápita, sumatorias, promedios, correlaciones) con el fin de visualizar la Seguridad Alimentaria en el Estado de Veracruz. Se analizaron datos correspondientes a los Pilares de la Seguridad Alimentaria: Producción, Utilización biológica y Accesibilidad, además de incluir indicadores Demográficos.

Se presenta también la descripción de los datos más relevantes encontrados en el Observatorio de Seguridad Alimentaria y Nutricional de interés para académicos, investigadores y tomadores de decisiones.

\section{Fuentes de información}

La información proviene de fuentes oficiales como es el caso de: Instituto Nacional de Estadística y Geografía (INEGI), Secretaría de Agricultura, Ganadería, Desarrollo Rural, Pesca y Alimentación (SAGARPA), Secretaría de Salud, Consejo Nacional de Evaluación de la Política de Desarrollo Social (CONEVAL), Secretaría de Desarrollo Social (SEDESOL).

\section{Resultados}

Indicadores demográficos en el Estado de Veracruz

La Vigilancia de la Seguridad Alimentaria y Nutricional (VSAN) se realiza por medio del análisis de indicadores, en el Estado de Vera- cruz esta vigilancia resulta compleja, en principio por la gran cantidad de municipios que lo componen (212) además de la diversidad cultural, climática, territorial, presencia del fenómeno migratorio, entre otros.

La población total para el año 2015 fue de 8,048,129; la Población Económicamente Activa aumentó durante el periodo de 2010 a 2015, pasando de 2,905,273 a 2,956,089, pero la población desocupada se duplicó, pasando de 106,137 habitantes a 276,868 habitantes, a este respecto es interesante considerar lo que señala Urquía (2014) mencionando la estrecha correlación existente entre la carencia alimentaria y el ingreso laboral.

Entre los fenómenos demográficos relevantes resalta el incremento en el número de nacimientos al pasar de 107185 para el año 2008 a 135584 para el año 2014. El número de hogares fue de 1,798,554 en 2005 mientras que para el 2015 fueron 2,251,217, de ellos, los hogares con jefatura femenina pasaron de 459,191 a 692,882 para el periodo comprendido de 2005 a 2015. En este aspecto, el Fondo de la Naciones Unidas para la Agricultura y la Alimentación, FAO (2013) encontró un aumento de 3 puntos porcentuales en hogares con carencia en acceso a la alimentación en los hogares que tienen jefatura femenina con respecto a los hogares con jefatura masculina, aunque también menciona que no hay datos contundentes respecto a la asociación de la jefatura femenina con condiciones de mayor precariedad.

\section{Producción de alimentos}

Los alimentos considerados en el OBSAN-UV son leche, carne y huevo como fuente de proteína de alta calidad, también se incluyeron maíz, arroz y frijol como productos básicos en nuestra dieta.

Al realizar comparaciones en la producción de alimentos, es importante destacar la disminución en la producción de leche, huevo, maíz y frijol, situación contraria a lo ocurrido con la carne para el periodo comprendido de 2010 a 2015.

1 Observatorio en Seguridad Alimentaria y Nutricional, Facultad de Nutrición - Xalapa. Contacto: lollopez@uv.mx 
A continuación, mencionaremos la producción per cápita para el estado de los alimentos ya señalados para el año de 2014.

- La producción per cápita de leche fue de 80.5792 litros, entre los municipios productores principales se encuentran Acajete, Playa Vicente, San Juan evangelista, Ozoluama y Naolinco .

- En cuanto a la Producción per cápita de huevo durante el año 2014, fue de 1.961 kilos siendo los principales municipios productores Sayula de Alemán, Hueyapan de Ocampo, San Juan Evangelista y Acayucan

- La Producción de maíz la producción per cápita para el Estado de Veracruz, fue de 120.051 kilos, destacando como principales productores los municipios de Soteapan, José azueta, Texistepec y Tatahuicapan

- La producción per cápita de Frijol fue de 2.2529 kilos, los municipios que produjeron mayor cantidad de este alimento fueron: Chicontepec, Perote, Benito Juárez, Soteapan y Hueyapan de Ocampo.

- En el caso del arroz, en el mismo año, la produccion per cápita es de 2.440 kilos, solo siendo productores en el Estado: Tierra Blanca, Tlalixcoyan, Tres Valles y Playa Vicente

- La producción per cápita de carne fue de 81.644 kilos, destacando los municipios de Las Choapas, Jalacingo, Cuitláhuac, Perote, Minatitlán y Tomatlán.

La disminución en la producción de maíz, frijol y arroz en México, durante el periodo comprendido de 1990 a 2011 ha sido documentada por la FAO (2013) lo cual cobra especial significancia al considerar lo señalado por Urquía (2014) quien menciona que Veracruz es uno de los siete estados que concentra el 50\% de la producción agrícola en el país.

\section{Accesibilidad}

El Pilar de accesibilidad nos proporciona un panorama sobre el acceso hipotético de la población a los alimentos, ya sea por capacidad adquisitiva o por programas sociales. En este rubro se encontró lo siguiente:

\section{Pobreza e índice de rezago social}

Los indicadores de pobreza a nivel municipal para el año 2015 no se encuentran disponibles a la fecha, la carencia estos datos complica en gran medida el análsis de este indicador; por lo anterior, hemos recurrido a utilizar el Índice de rezago social, que se considera como una medida aproximada, para medir la pobreza.

De acuerdo con CONEVAL, el índice de rezago social es una medida ponderada que resume cuatro indicadores de carencias sociales (educación, salud, servicios básicos y espacios en la vivienda) es un índice que tiene como finalidad ordenar a las unidades de observación según sus carencias sociales. A nivel nacional, Veracruz ocupó para el año 2015 el cuarto lugar en Índice de rezago social, en el cuadro 1 podemos observar los municipios Veracruzanos que presentan mayor índice de rezago social.

Es notable que el índice de rezago social de estos municipios está muy por encima del promedio del Estado, situación preocupante al considerar que Veracruz es un Estado con alto índice de rezago social con relación al resto del país.

\section{Programas sociales}

El programa PROSPERA presentó una disminución en el número de beneficiarios con respecto al año 2011 en el cual operaba el Programa Oportunidades, sin embargo los Programas Liconsa (número de beneficiarios), Diconsa (número de tiendas) y el Programa 70 y mas (número de beneficiarios) aumentaron en su cobertura hablando de números absolutos..

Al realizar correlaciones, se pudo contatar que el índice de rezago social y Programa 70 y mas tienen una correlacióm de -0.33, indicando una correlación negativa entre los municipios con mayor rezago social y los beneficiarios al programa, siendo mas significativo esta correlación en los municipios en Tehuipango, Mixtla de Altamirano, Texcatepec, Zontecomatlán e Ilamatlán. 
Cuadro 1. Municipios del Estado de Veracruz con mayor índice de Rezago social en el año 2015.

\begin{tabular}{|c|c|}
\hline Nombre del municipio & Índice de rezago social \\
\hline Estado de Veracruz & 1.19939 \\
\hline TEHUIPANGO & 3.71904 \\
\hline MIXTLA DE ALTAMIRANO & 3.575617 \\
\hline ILAMATLAN & 2.44225 \\
\hline ZONTECOMATLAN & 2.42190 \\
\hline TEXCATEPEC & 2.38220 \\
\hline SOLEDAD ATZOMPA & 2.19343 \\
\hline ATLAHUILCO & 2.17384 \\
\hline TEQUILA & 2.17269 \\
\hline LOS REYES & 2.15028 \\
\hline ASTACINGA & 2.11914 \\
\hline TLAQUILPAN & 2.06530 \\
\hline
\end{tabular}

Fuente: CONEVAL 2015

Figura 1: Coeficiente de correlación r entre Índice de rezago social y número de beneficiarios del programa 70 y más: -0.33 (2015).

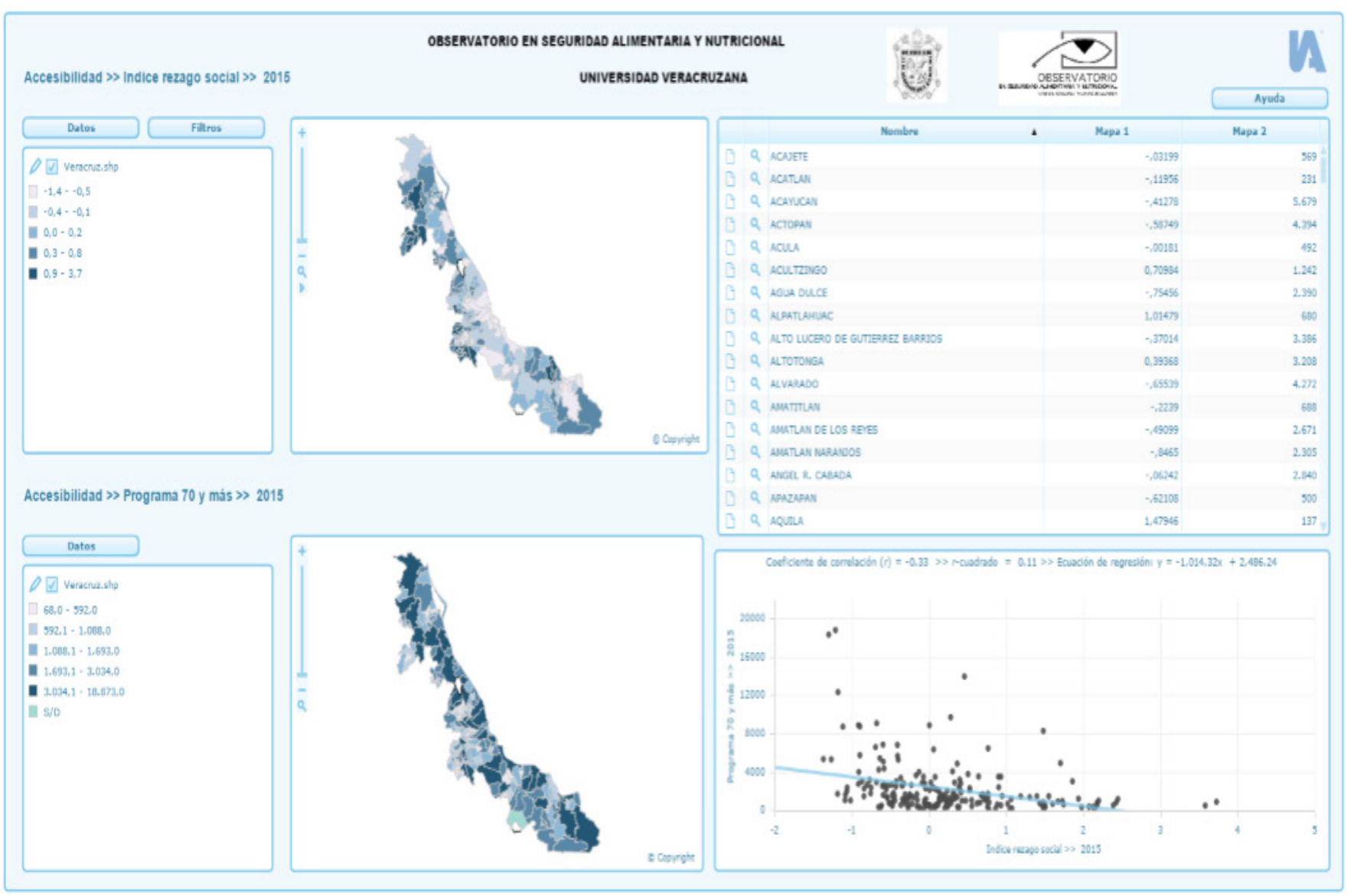

Fuente: OBSAN- UV 2017 
De igual manera en el análisis de correlación entre el índice de rezago social y el programa alimentario es 0.14 , habiendo municipios con un muy alto índice de rezago social y pocos beneficiarios con el programa alimentario, como es el caso de Tehuipango y Mixtla de Altamirano, que presentan índices de rezago social de 3.719039 y 3.575617 respectivamente y donde apenas un 3.4 y 6.5\% de su población son beneficiarios con el programa alimentario, lo cual evidencia una inadecuada planificación con enfoque de riesgo de este programa.

\section{Utilización biológica}

El pilar de Utilización Biológica se refiere al estado de salud de las personas, considerando que la alta incidencia de enfermedades relacionadas con la ingesta de alimentos, esto evidencia una pobre utilización biológica de los alimentos y sus nutrientes, que traen como consecuencia malnutrición e inseguridad alimentaria.

Cabe destacar que para el año 2015 en los niños de o a 4 años de edad con desnutrición y en los menores de 19 años con obesidad, se pre- senta un coeficiente de correlación de r: 0.62, evidenciado la coexistencia de desnutrición y obesidad para estos grupos de edad. Los municipios que presentan este fenómeno son: Martínez de la Torre, Xalapa, San Andrés Tuxtla, Xico y Veracruz, esta situación coincide con lo reportado a nivel Nacional por Urquía (2014).

Otras correlaciones destacadas son las que se dan entre índice de rezago social y el porcentaje de niños con desnutrición ( 0.25), es decir es posible que el índice de rezago social esté impactando en la prevalencia de desnutrición. En cuanto a la correlación $r$ que presentan índice de rezago social y obesidad en menores de 19 años es -0.28

Por su parte las infecciones respiratorias agudas (IRAS) en niños de 0-4 años, disminuyeron para el año de 2015 con respecto a 2007 cifras que correspondieron a 35,535 caos y a 154,839 casos respectivamente. Con respecto a los casos detectados con VIH para el año 2008 se reportaron 682 casos, cifra que se incrementó considerablemente para el año 2015 al reportarse 1147 nuevos casos.

Figura 2: Coeficiente de correlación r entre Índice de rezago social y Programa de apoyo alimentario: 0.14 (2015).

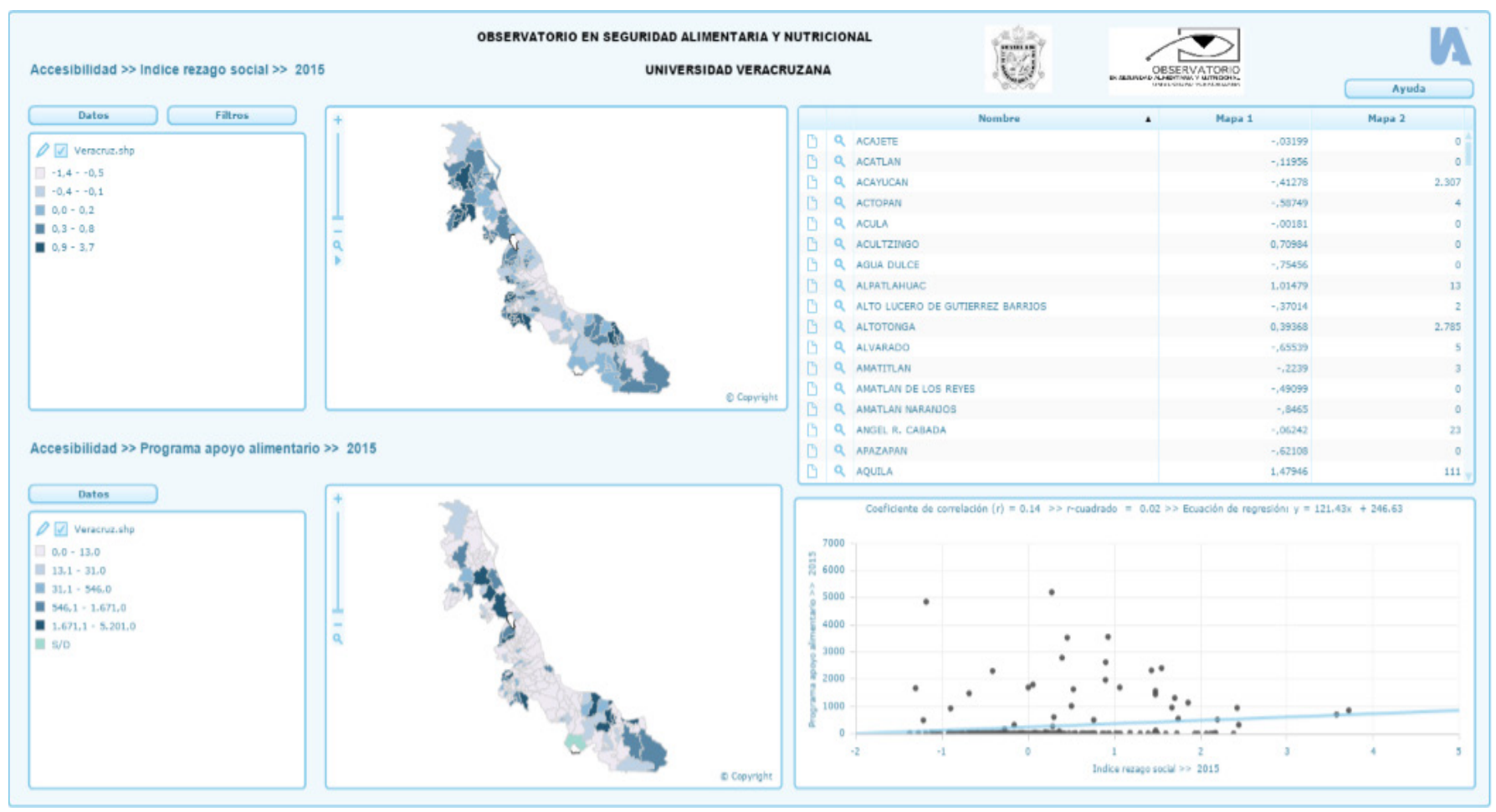

Fuente: OBSAN- UV 2017 
Figura 3: Coeficiente de correlación r entre niños de 0-4 años con desnutrición y menores de 19 con obesidad para el año 2015

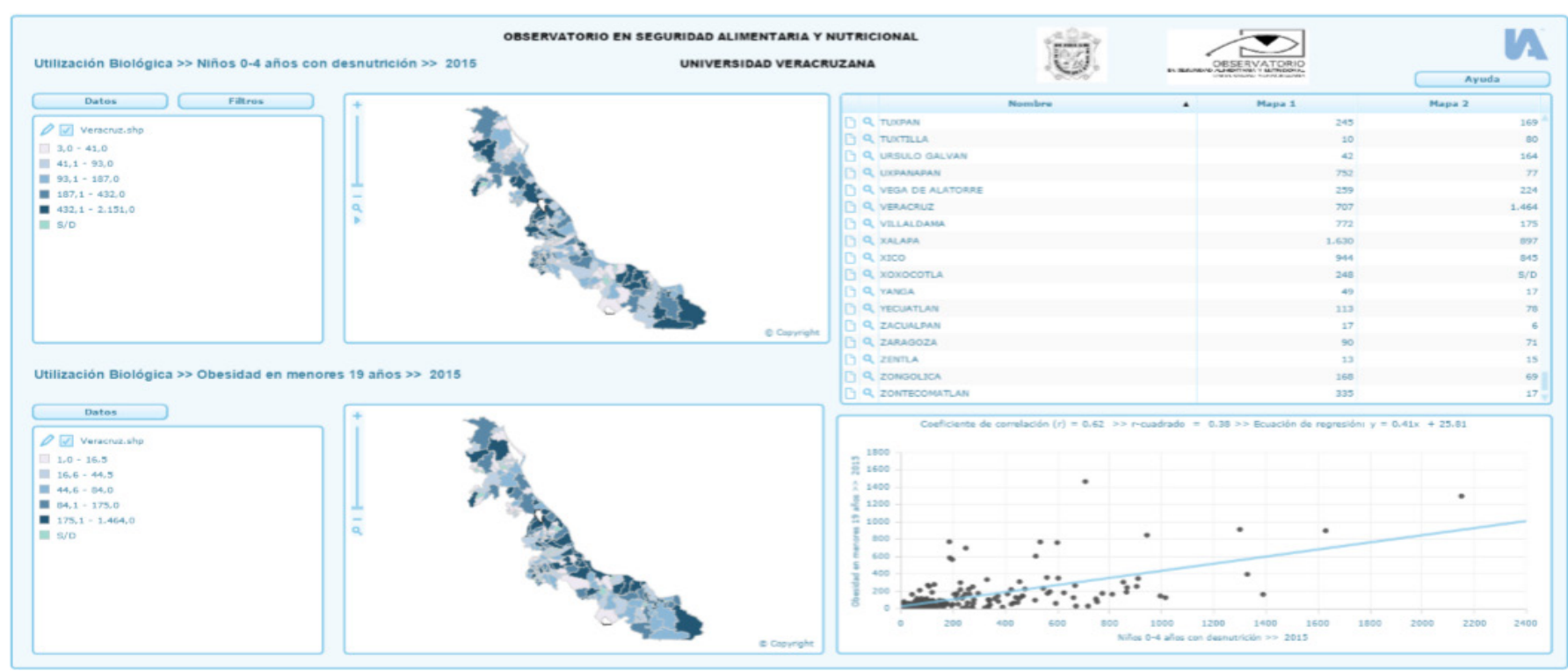

Fuente: OBSAN- UV 2017

Conclusiones

A través del análisis de algunos indicadores disponibles en el Observatorio en Seguridad Alimentaria y Nutricional en el Estado de Veracruz de la Facultad de Nutrición Xalapa de la Universidad Veracruzana se puede concluir lo siguiente:

- Algunos indicadores como es el caso de la muy baja producción per cápita de alimentos, población desocupada y el Muy alto índice de rezago social a nivel estatal y en algunos municipios evidencian la inseguridad alimentaria y nutricional.

- Los Programas de Asistencia Alimentaria no han logrado el impacto esperado en la población beneficiaria, pero estos tampoco benefician a la población más vulnerable.

- Es importante considerar políticas púbicas que activen la economía del estado favoreciendo la producción y disponibilidad de alimentos

- Al realizar un análisis de la correlación existente entre desnutrición y obesidad en la población de menores de 19 años, se pudo constatar la polarización de los problemas nutricionales en donde cohabitan la desnutrición y la obesidad.

- Los casos de IRAS en niños de 0-4 años de edad disminuyeron un 74\% de 2007 a 2015, sin embrago los casos de VIH aumentaron, por lo que la demanda social de la enfermedad infecciosa demanda un incremento en gasto público asignado a la atención a la salud.

\section{Referencias}

Universidad Veracruzana (2017). Observatorio en Seguridad Alimentaria y Nutricional. Recuperado de: https:// www.uv.mx/obsan/

Food and Agriculture Organization of the United Nations, (2016). Monitoreo de la seguridad alimentaria y nutricional como apoyo a políticas públicas en América Latina y el Caribe. Santiago de Chile. Recuperado de: http:// wWw.fao.org/3/a-i5442s.pdf

FAO. Panorama de la seguridad alimentaria y nutricional en México 2012. Informe país. México: FAO, Sagarpa, Sedesol, Coneval, INSP, 2013.

Urquía-Fernández, Nuria. (2014). La seguridad alimentaria en México. Salud Pública de México, 56(Supl. 1), s92-s98. Recuperado el 31 de enero de 2018, de http:// www.scielo.org.mx/scielo.php?script=sci_arttext\&pi$d=S 0036-36342014000700014 \& \operatorname{lng}=$ es\&tlng $=$ pt. 